\title{
Study on Worldwide Development and Trends of Quantum Technologies Based on Patent Data
}

\author{
Juan Zhang, Qianfei Tian, Chuan Tang, Lina Wang, Jing Xu, and Junmin Fang
}

\begin{abstract}
Quantum technologies attracted much attention for their disruptive potential in last two decades. The article analyzes the worldwide patent landscape for quantum technologies based on data extracted from Derwent Innovation and Web of Science. The quantum technologies were grouped into three distinct technology areas of quantum computing, quantum communication and quantum sensing, to demonstrate detailed development and trends respectively. It shows that quantum technology is a highly competitive research field, and United States, China and Japan are the most prominent countries, in particular China made a great progress in recent years. United States has a significant advantage in the field of quantum computing, which is the most promising field, meanwhile China has a significant advantage in the field of quantum communication and succeeds in launching a quantum satellite.
\end{abstract}

Index Terms-Patent analysis, quantum communication, quantum computing, quantum sensing.

\section{INTRODUCTION}

In the recent two decades, quantum technologies, which make use of the "strange" quantum properties, such as superposition and entanglement [1], have attracted much attention in social circles, for their breakthrough applications expected in space, energy, environment, security, health, and scientific research of diverse disciplines. A lot of countries have launched large research programmes on quantum technologies. For example, the US "National Quantum Initiative Act" [2] signed into law established a coordinated multiagency National Quantum Initiative Program to accelerate the development of quantum information science and its technology applications; European quantum technology flagship initiative with $€ 1$ billion budget was launched to develop a quantum web, where quantum computers, simulators and sensors are interconnected via quantum communication networks [3]; the UK national strategy for quantum technologies [4] and the accompanying roadmap [5] aimed at creating a coherent government, industry and academic quantum technology community; China launched a quantum satellite Micius and accomplished a quantum key distribution (QKD) link spanning Europe and Asia [6]; Germany [7] and Japan [8] also developed ambitious plans for quantum technologies. Meanwhile, large multinational corporations like IBM, Intel, Google, Microsoft, Toshiba, NEC etc., have started to invest heavily in quantum technologies, a number of start-ups were

Manuscript received June 14, 2019; revised Janaury 15, 2020.

The authors are with Chengdu Library and Information Center, Chengdu, Sichuan, China (e-mail: zhangj@clas.ac.cn, tqf@clas.ac.cn, tangc@clas.ac.cn, wangln@clas.ac.cn,jingxu@clas.ac.cn, fjm@clas.ac.cn). established as well.

Quantum technologies can be grouped into three categories: quantum computing \& simulation, quantum communication, quantum sensing and measuring [9]. Quantum computing can dramatically speed up certain complex problem-solving that would otherwise take billions of years for today's classical computers. Quantum communication can protect information channels against eavesdropping by means of quantum cryptography. Quantum sensing can be exploited to enhance the performance of measurements of physical quantities, atomic clock is an example of quantum sensor.

Patent data can give a valuable insight into technology development and trends, then the article analyzes the worldwide patent landscape for quantum technologies, focusing on quantum computing (including quantum simulation), quantum communication and quantum sensing (including quantum measuring and timing) respectively, in order to give a comprehensive overview of quantum technologies, as well as their development and application situation and trends, which may help for the planning and investment in quantum technology research in future.

\section{Methodology}

The data used for analysis were extracted from the worldwide patent database Derwent Innovation (DI), then were cleaned and treated by Derwent Data Analyzer (DDA) and DI. The quantum technologies fall into three distinct technology areas of quantum computing, quantum communication and quantum sensing. At the same time, the brief bibliometric analysis based on Web of Science for quantum technologies was carried to compare with the patent analysis, in order to show unique features of development of quantum technologies.

\section{A. Data Retrieving and Cleaning}

Searching was conducted in Derwent Innovation and Web of Science, for the concepts of quantum computing, quantum communication, quantum sensing respectively. The key words for searching included "quantum comput*", "quantum chip(s)", qubit, "quantum simulation", "quantum communicat*", "quantum key distribution", "quantum sens*", "quantum measure*", and so on. Patent collection was limited to one document per family using Derwent World Patent Index (DWPI) families. Assignee names were standardized based on known mergers, acquisitions, and change of ownership. There were 2631 documents for quantum computing, 1732 for quantum communication and 1180 for quantum sensing based on the patents searching by 
June $3^{\text {rd }}, 2019$.

\section{B. Data Analysis and Visualization}

DDA and DI were used to clean and treat the raw data, as well as data integration and visualization. We studied the yearly publication or application trends, life cycle of quantum technologies, as well as major countries, core institution and key research areas in quantum computing, quantum communication and quantum sensing $R \& D$ respectively, to show the development at the moment and the trends in future.

\section{OVERVIEW OF DEVELOPMENT OF QUANTUM TECHNOLOGIES}

\section{A. Overall Development Trends and Features}

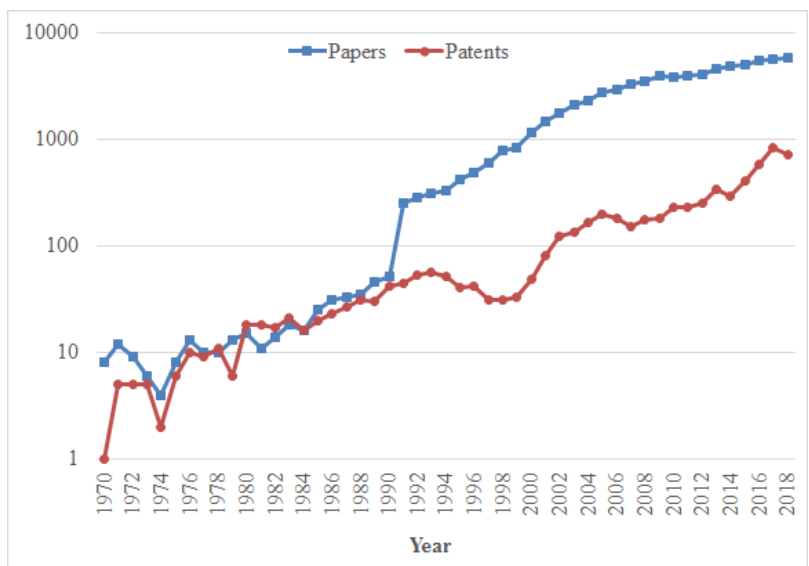

Fig. 1. Overall quantum technologies development trend based on publication by year.

(Note: the patent application publishing has almost 18-month delay from their earliest filing date, so there may have not the whole patent data in 2018 and 2019.)

As shown in Fig. 1, quantum technologies have been seen rapid growth over the last thirty years, based on both patent application and paper publication. In order to provide more details about overall development trend of quantum technologies, we changed the scale of the $\mathrm{Y}$ axis to logarithmic. The paper publication related to quantum technologies stepped to a new level from 1991 and maintained a quick increase since then. Meanwhile, the progress of patent application was stagnating and declined to a low stage in the latter half of 1990s. Since 2002, the patent application of quantum technologies moved up a gear, attributing largely to more and more attraction that quantum computing and quantum communication got. It seems that the surging of theory researches about quantum technologies attracted attention to their disruptive potential application and promoted the technology transfer. The patent application moved up to another notch and showed extraordinary growth since 2014, in line with the boom in commercialization of quantum technologies from 2014. At the same time, more and more governments and corporates produced ambitious plans and made huge investments to quantum computing, quantum communication and quantum sensing, the institutions and personal researchers also put a lot of effort as well.

Fig. 2 showed overall development trends of three different quantum technologies. As shown in Fig. 2(a), there was a quick increase in the patent application of three quantum technologies over the last five years, the same as Fig. 1. For quantum computing, there was a jump in patent application in 2002 that continued with steady growth through 2014, then showing an exponential growth from 2014, which can be verified by the large scale investment from transnational companies, like Google, IBM, Microsoft, Intel, D-Waves, etc., as well as a number of governments. The field of quantum communication demonstrated the same trends as quantum computing, but the onset of the former was over 20 years later than the latter. The research of quantum sensing began in 1960s and made slower progress compared with the other two. Fig. 2(b) showed the academic paper publication trends by year, the field of quantum computing showed a much steeper increase than the other two, giving the facts that quantum computing is in spotlight and attracting much more attention whether for its prospect in application, or the theory behind technology.

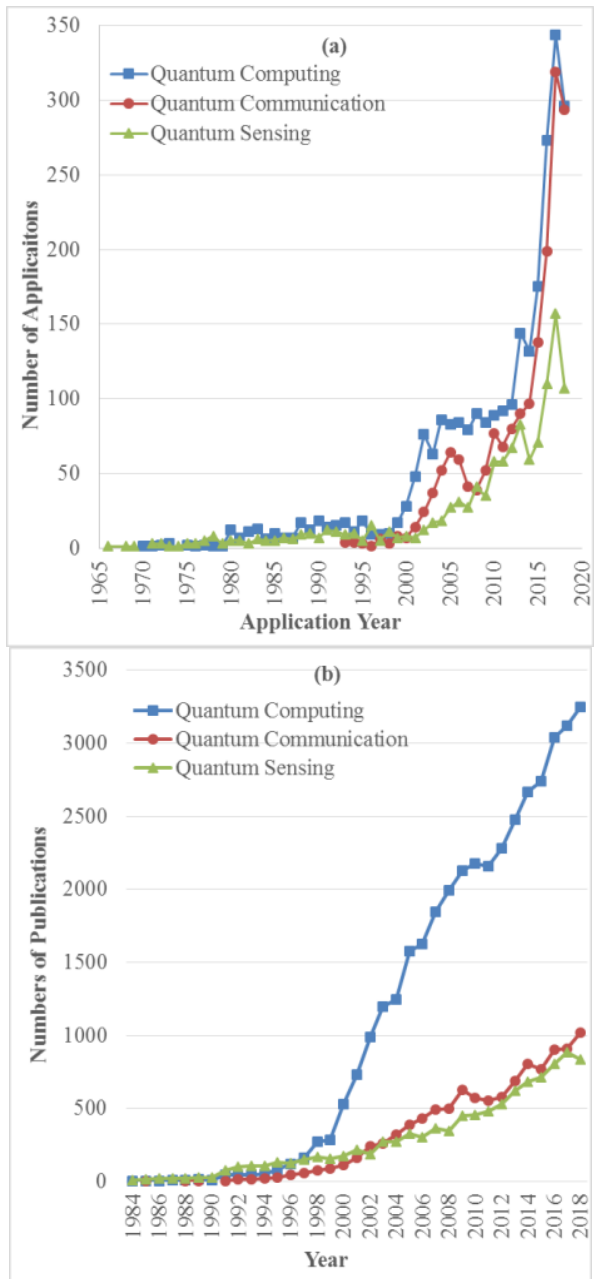

Fig. 2. Overall development trends for different quantum technologies by year (a) patent application; (b) paper publication.

\section{B. Technology Life Cycle Analysis}

The life cycle of quantum technologies based on patent analysis was shown in Fig. 3. Before 2000, all of three quantum technologies were in the period of technology trigger, with a few of both application documents and assignees / applicants. From 2000 or so, quantum technologies entered the period of development. For the field of quantum computing, more and more institutions and 
companies took part in, showing rapid growing of assignees since 2002, which contributed to the first wave of growth as showed in Fig. 1 and Fig. 2. But as the numbers of application were stable in this period, this growth was smooth. For the field of quantum communication, there also had a steady growth because both assignees and applications were showed moderate growth, which is different from the field of quantum computing. Both quantum computing and quantum communication entered the period of rapid expansion since 2014, with the rapid growth of assignees and applications. Quantum computers with different qubit roadmap appeared with increasing of numbers of qubits, quantum communication network was established and quantum satellite was launched, more and more start-ups emerged as well. The field of quantum sensing entered the period of development since 2003 and remained relatively rapid growth, except for a little stagnation from 2010 to 2014. The application increased quickly against a slow increase of assignee since 2015, which indicated that the quantum sensing technology is tending to mature, showing a different trends with the other two.

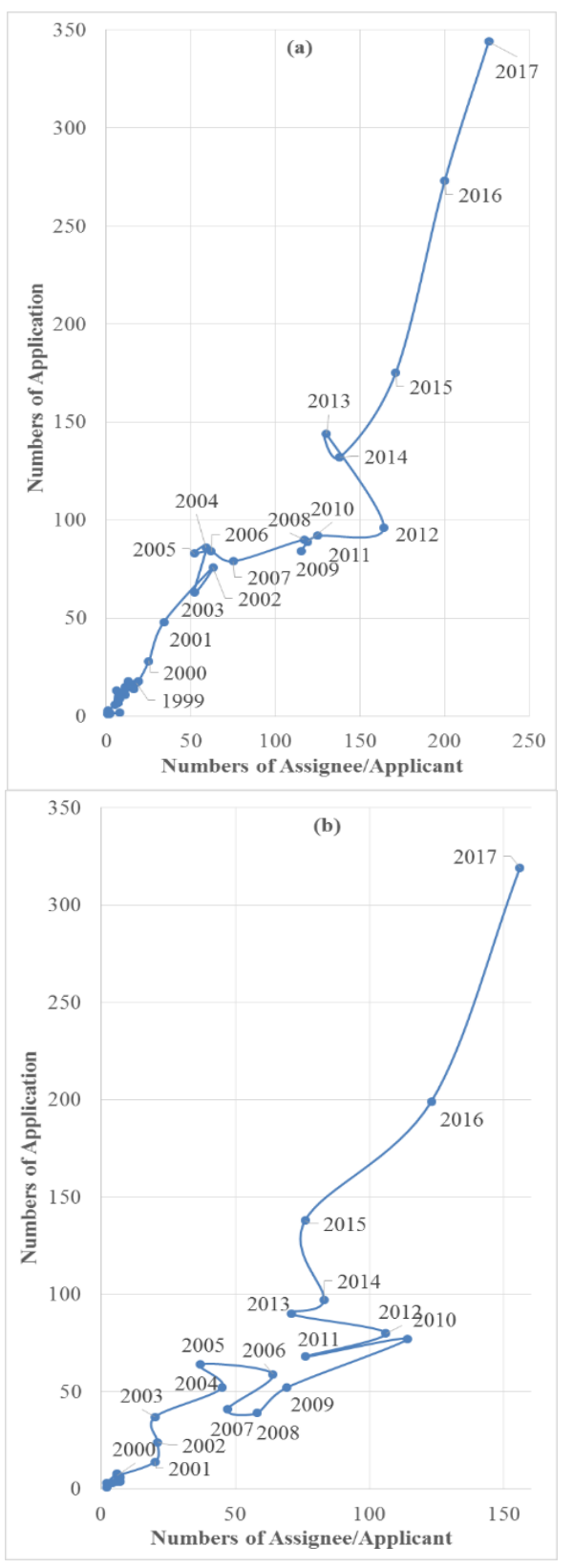

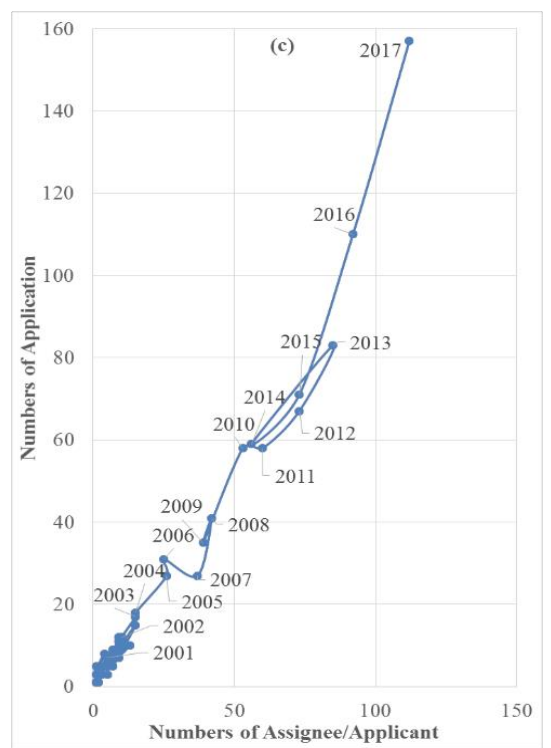

Fig. 3. Life cycles for quantum technologies: (a) quantum computing; (b) quantum communication; (c) quantum sensing.

\section{DEVELOPMENT AND TRENDS FOR QUANTUM TECHNOLOGIES BY CATEGORIES}

\section{A. Quantum Computing}

As shown in Fig. 4, in the field of quantum computing, the United States was the most prominent country with 1170 priority filings, surpassing the sum of Japan, which was second with 545 priority filings, and China, which was third with 520 priority filings. United States also remained high speed growth of patent application and publishing. Japan reached the peak around 2004 and was in decline after then. China started quantum computing research on 2003 in the view of patent application, much later than United States and Japan, but made a great progress and had a steep growth in patent application recent years.

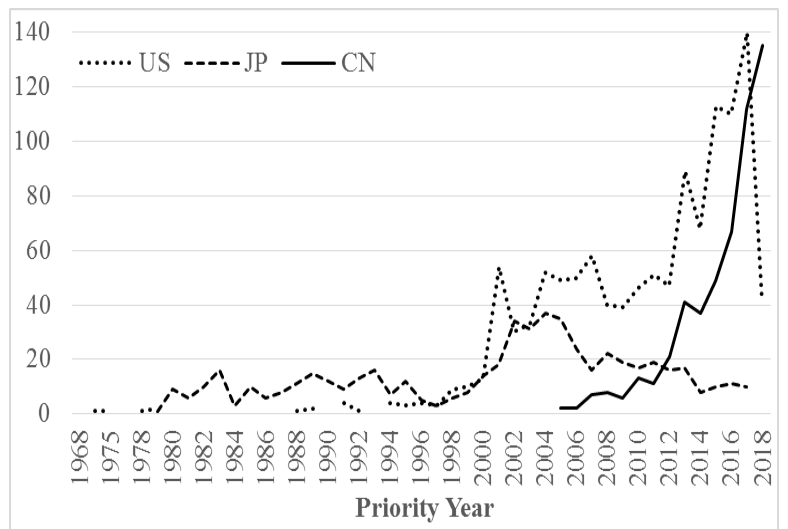

Fig. 4. Quantum computing development trends based on patent by priority country and priority year.

Fig. 5 showed Top 20 assignees in quantum computing field, which illustrated that the quantum computing $R \& D$ was carried mainly by companies. D-Wave from Canada had the largest portfolio of quantum computing patent families in this study, Hefei Origin Quantum from China made large progress and ranked No. 15. United States and Japan each had nine of these core companies and institutions, showing their powerful and solid strength in research of quantum computing. 


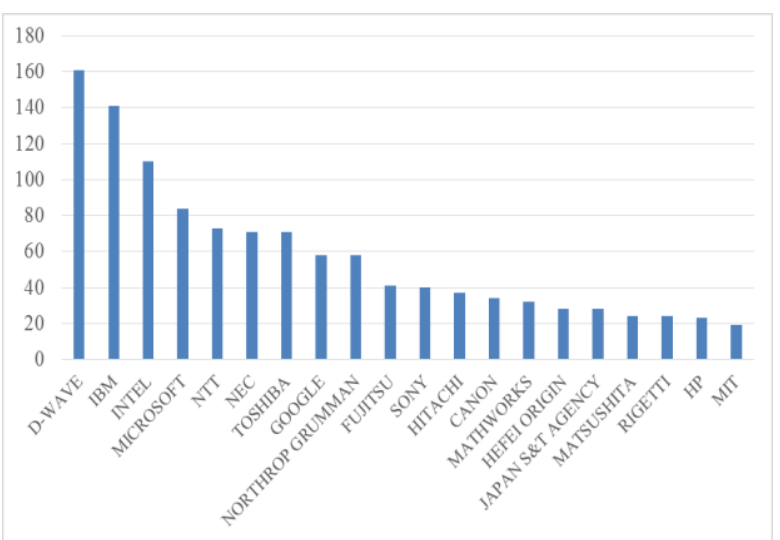

Fig. 5. Top 20 assignees in quantum computing R \& D.

Fig. 6 showed the core technologies for quantum computing and the layout of United States, China and Japan in this field. The research and application of quantum computing focused on the areas of logical qubits, quantum devices, superconductor materials, image and video, key etc., which may be grouped in three parts of qubit, device or hardware, and application (including algorithm). United States developed overall planning for all of three areas, with particular interest in qubit and device, where the multinational corporations like Google, IBM, Intel, and Microsoft heavily invested on. China had the greatest concentration in the area of application, with particular emphasis on image data and algorithm. Japan concentrated on application, especially for signal quantization, but also put emphasis on semiconductor device.

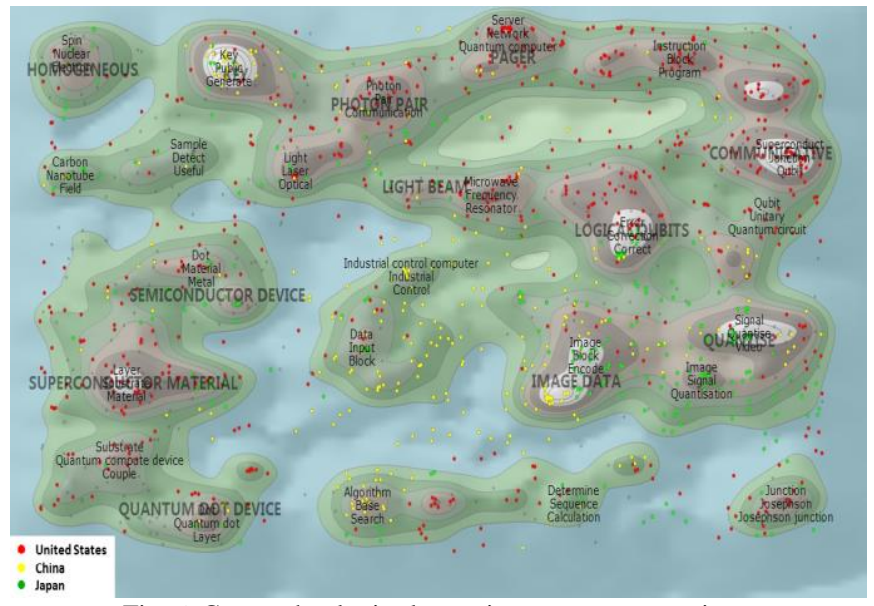

Fig. 6. Core technologies layout in quantum computing.

\section{B. Quantum Communication}

As shown in Fig. 7, China had a significant advantage in the field of quantum communication to outshine others with 955 priority filings, more than half of all filings. United States gave rise to a small upsurge in quantum communication in the first half of 2000s. It can be seen that United States and Japan pay little attention to quantum communication at present.

Top 20 assignees in quantum communication field were shown in Fig. 8, most of them were companies. There were 13 Chinese assignees, 5 of which were universities or academic institutes. In the rest, three came from Japan, two came from Korea, one came from United States, and one came from Malaysia.

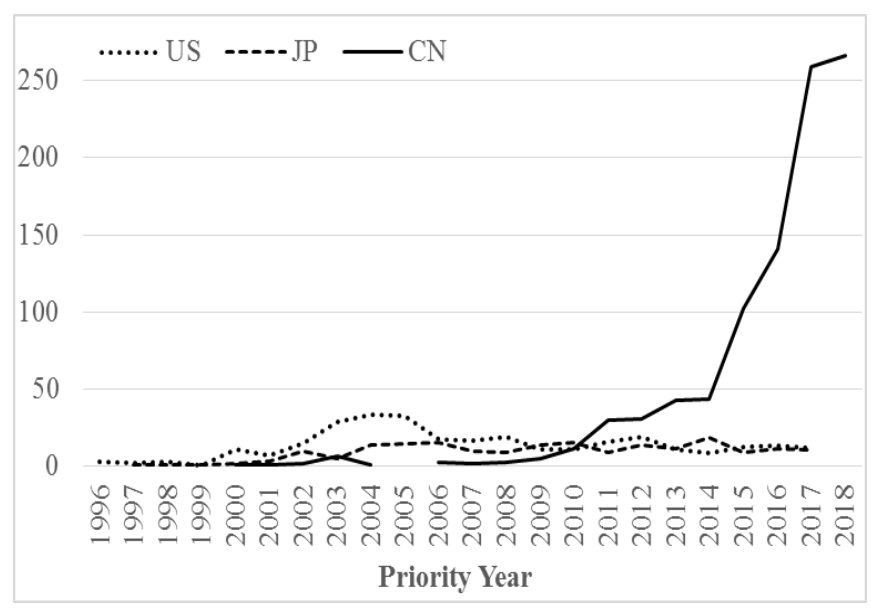

Fig. 7. Quantum communication development trends based on patent by priority country and priority year.

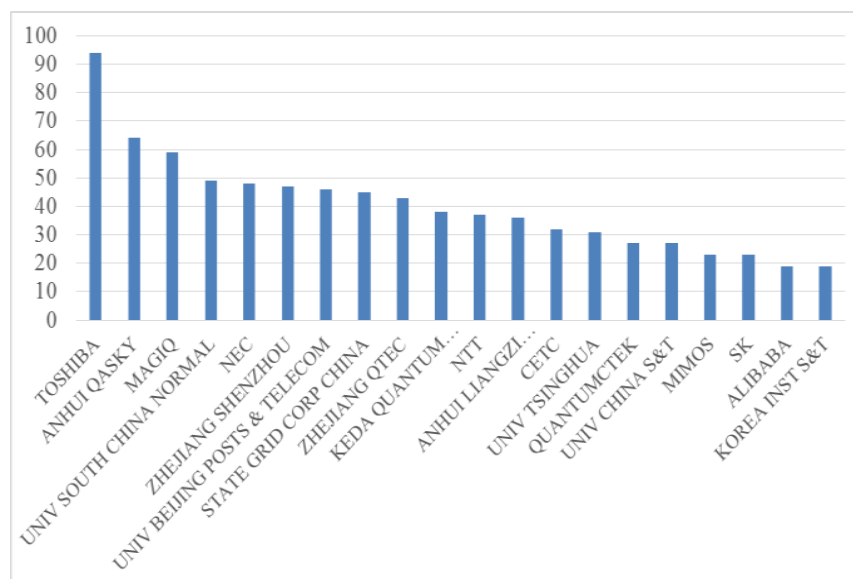

Fig. 8. Top 20 assignees in quantum communication R \& D.

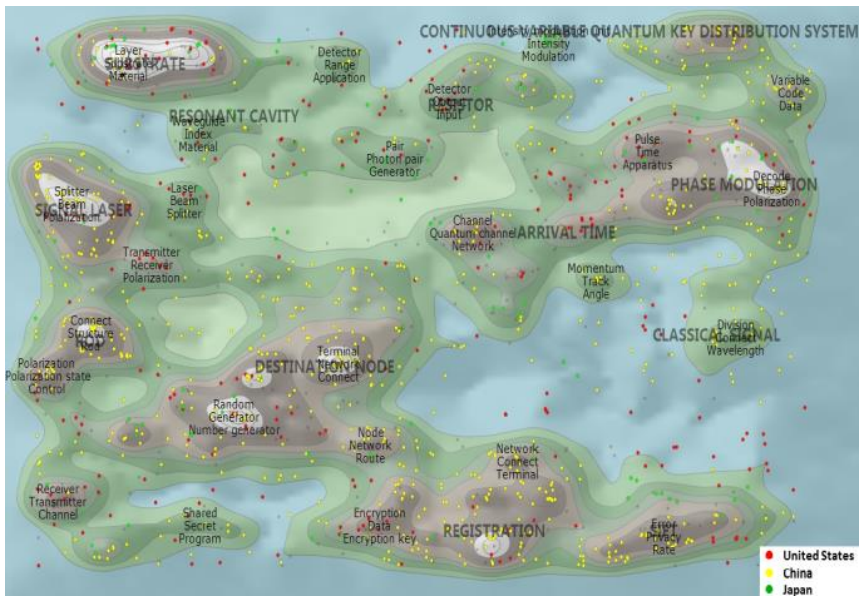

Fig. 9. Core technologies layout in quantum communication.

Fig. 9 showed the core technologies for quantum communication and the layout of United States, China and Japan in this field. The research and application of quantum communication focused on the areas of quantum key distribution, phase modulation, signal laser, quantum channel, random generator, number generator, polarization, encryption, registration, resistor etc. Chinese research in quantum communication covered a variety of technology topics, which made it take the leading position in this field.

\section{Quantum Sensing}

In the field of quantum sensing, China, United States and Japan were still Top 3 countries with 491, 351 and 153 
priority filings respectively, particularly China remained a very high rate of rise in last three years, as show in Fig. 10. In general, attention that quantum sensing drew was not as much as quantum computing and quantum communication. From another point of view, we could say quantum sensing may be the most mature technology among the three quantum technologies.

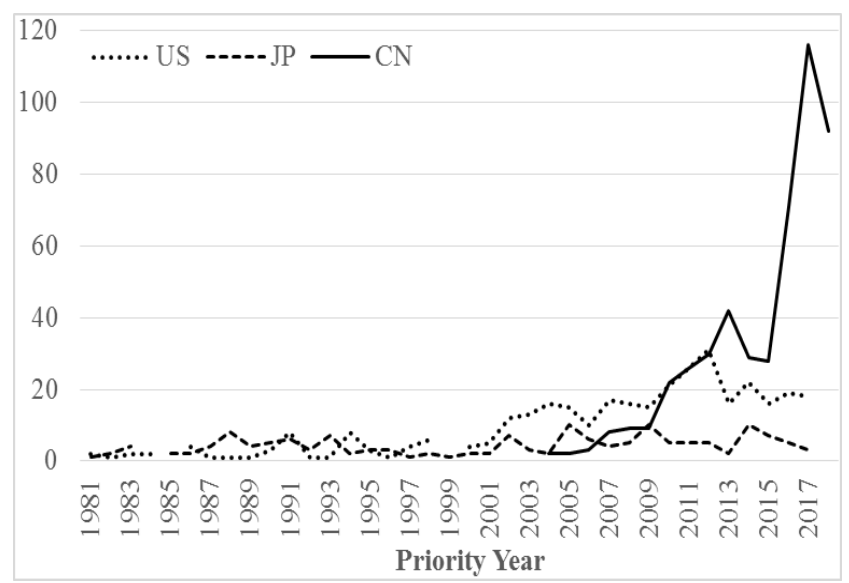

Fig. 10. Quantum sensing development trends based on patent by priority country and priority year.

Top 20 assignees in quantum sensing field were shown in Fig. 11, half of them were academic institutes or universities, most of which came from China. In all assignees, 11 came from China, 4 came from United States, 4 came from Japan, and one came from Switzerland. The assignees from United States and Japan were all companies.

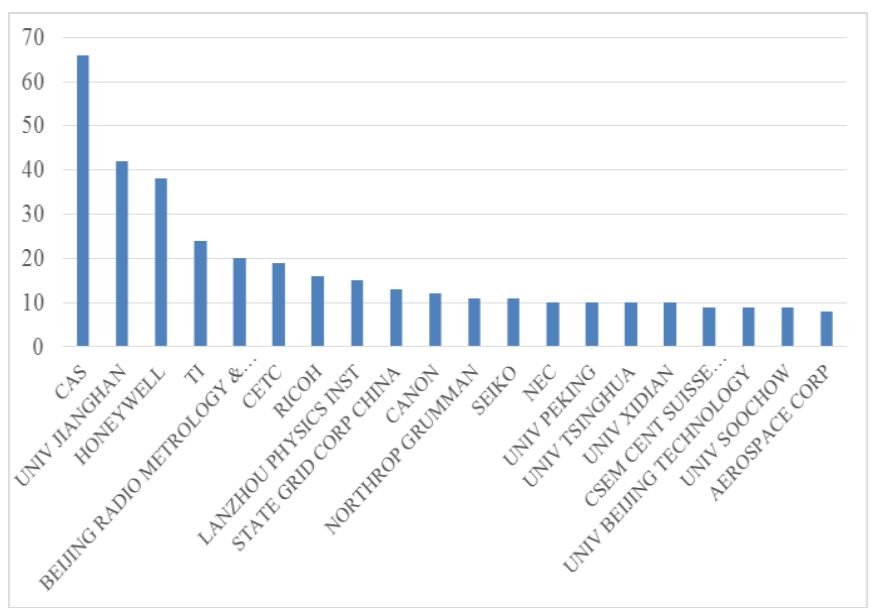

Fig. 11. Top 20 assignees in quantum sensing R \& D.

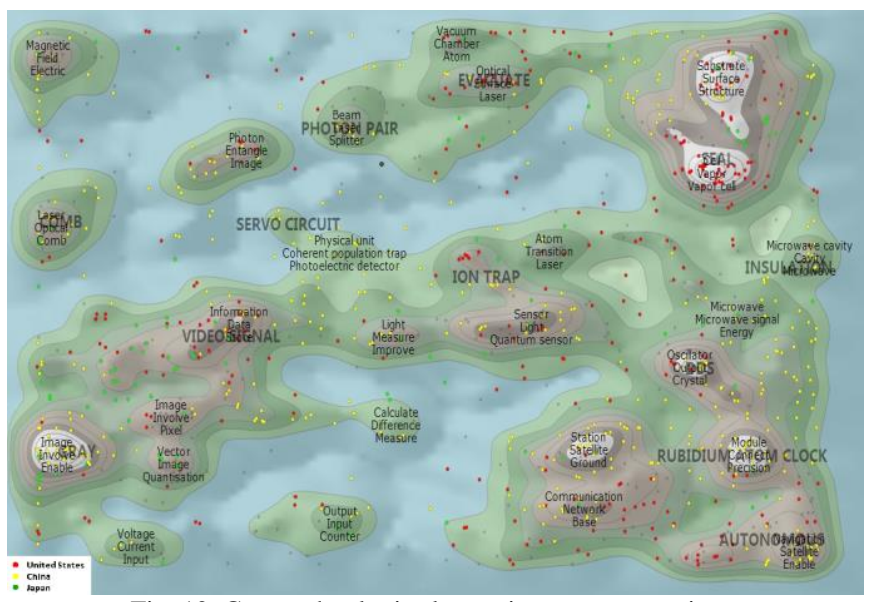

Fig. 12. Core technologies layout in quantum sensing.
The core technologies for quantum sensing and the layout of United States, China and Japan in this field were shown in Fig. 12. The research and application of quantum sensing focused on the areas of substrate surface structure, vapor cell, rubidium atom clock, quantum sensor, ion trap, servo circuit, photo pair, video signal, evacuate, autonomous etc. Both of China and United States developed overall planning for quantum sensing $\mathrm{R} \& \mathrm{D}$, involving a variety of key technologies. Japan concentrated on video signal, image and information store.

\section{CONCLUSION}

Based on above results and discussion, the article give an overall picture for the evolution of quantum technologies, as well as detailed trends analysis for quantum computing, quantum communication and quantum sensing respectively. Quantum technologies show an exponential growth in recent years, especially for quantum computing, for it disruptive potential to change traditional computation and widespread scientific research. Hence transnational corporations such as Intel, Google, IBM, and Microsoft compete fiercely for quantum computing, as well as many governments. Quantum communication has also attracted much interest and gave some successful cases for application, but there still need a lot of endeavor to achieve quantum web. Quantum sensing is getting more mature and close to practice, which has been pointed out in UK's roadmap for quantum technologies: a number of application for quantum sensing are expected to arise within 5 years, meanwhile, it is expected to be 20 years at least before the practical quantum computers are widely available [5].

For countries, Unites States, China and Japan have significant advantages in quantum technologies $R \& D$, particularly China made a great progress in recent years, for example, quantum satellite Micius launched. United States occupies a position of strategic importance in quantum computing research and develops an overall planning. China lacks in strength for core technologies $R \& D$ of quantum computing, such as research for qubit technology and quantum devices. Japan progresses steadily and focuses on application of quantum technologies as usual.

\section{CONFLICT OF INTEREST}

The authors declare no conflict of interest.

\section{AUTHOR CONTRIBUTIONS}

Juan Zhang (zhangj@clas.ac.cn, corresponding author) proposed the research idea, designed the research, drafted and revised the manuscript. Qianfei Tian (tqf@clas.ac.cn) conducted the research and revised the manuscript. Chuan Tang (tangc@clas.ac.cn) collected and analyzed the data. Lina Wang (wangln@clas.ac.cn) analyzed and visualized the data. Jing Xu (jingxu@clas.ac.cn) analyzed and visualized the data. Junmin Fang (fjm@clas.ac.cn) provided useful advice for the research and revised the manuscript, and all authors had approved the final version. 


\section{REFERENCES}

[1] A. Acín, I. Bloch, H. Buhrman et al., "The quantum technologies roadmap: A European community view," New J. Phys, vol. 20, pp. 1-24, August 2018.

[2] American Institute of Physics. (December 2018). National Quantum Initiative Act - H.R.6227 / S.3143. [Online]. Available: https://www.aip.org/fyi/federal-science-bill-tracker/115th/national-qu antum-initiative-act

[3] European Commission. (October 2018). Quantum Technologies Flagship Kicks off with First 20 Projects. [Online]. Available: http://europa.eu/rapid/press-release_IP-18-6205_en.htm

[4] UK Government. (March 2015). National Strategy for Quantum Technologies. [Online]. Available: https://www.gov.uk/government/publications/national-strategy-for-qu antum-technologies

[5] UK Government. (October 2015). A roadmap for quantum technologies in the UK. [Online]. Available: https://www.gov.uk/government/uploads/system/uploads/attachment_ data/file/470243/InnovateUK_QuantumTech_CO004_final.pdf

[6] Livescience. (January 2018). China's Quantum-Key Network, the Largest Ever, Is Officially Online. [Online]. Available: https://ww.w.livescience.com/61474-micius-china-quantum-key-inter continental.html

[7] BMBF. (September 2018). Quantum technologies -from basic research to market. [Online]. Available: https://www.bmbf.de/upload_filestore/pub/Quantum_technologies_fr om_basic_research_to_market.pdf
[8] JST. (January 2019). MEXT - Quantum Leap Flagship Program (MEXT $Q$-LEAP). [Online]. Available: https://www.jst.go.jp/stpp/q-leap/en/index.html

[9] G. Kalbe. (June 2016). The New Flagship on Quantum Technologies State of Play. [Online]. Available:
S https://www.flagera.eu/wp-content/uploads/2016/02/05-EC-QT-Flags hip-State-of-Play-30-June-2016.pdf

Copyright (C) 2020 by the authors. This is an open access article distributed under the Creative Commons Attribution License which permits unrestricted use, distribution, and reproduction in any medium, provided the original work is properly cited (CC BY 4.0).

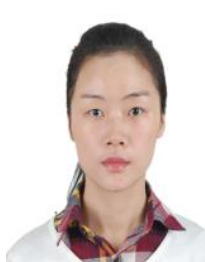

Juan Zhang was born Chengdu city, Sichuan province, China, on Aug. 24, 1979. She got the Ph.D in the major of polymer materials, from the Department of Natural Science and Ecological Awareness, Nara Women's University, Japan in 2009. She is working at Chengdu Library and Information Center, CAS. Since the year of 2009, and has visited Indiana University in the first half year of 2015. Now she is an associate professor with study on scientometrics, informetrics and data mining, as well as strategic information analysis in the area of information and communication technology, using both qualitative and quantitative method, including policy study, numerical data analysis, bibliometrics, visualization analysis etc. 